\title{
Gender-Affirming Telepsychology During and After the COVID-19 Pandemic: Recommendations for Adult Transgender and Gender Diverse Populations
}

\author{
Tyson L. Pankey · Dagoberto Heredia Jr · Jennifer A. Vencill · Cesar A. Gonzalez
}

Published online: 16 October 2021

(c) National Register of Health Service Psychologists 2021

\begin{abstract}
The implementation of telepsychology soared in response to the 2019 novel coronavirus (COVID-19) pandemic. For most health service psychologists, this surge preceded formal training in telepsychology. Transgender and gender diverse (TGD) individuals reported significant vulnerabilities and health disparities during the COVID-19 pandemic. To ensure the health and well-being of adult transgender and gender diverse individuals during the COVD-19 pandemic and beyond, it is critical to promote the delivery of gender-affirming telepsychology. This article highlights clinical issues observed by health service psychologists at a high-volume gender clinic during the COVID-19 pandemic. The authors provide anticipatory guidance and recommendations to promote gender-affirming telepsychology.
\end{abstract}

Keywords telehealth $\cdot$ telepsychology $\cdot$ transgender health $\cdot$ gender-affirming care

\section{Clinical Vignette}

A primary care clinician referred a 25 -year-old to you because of concerns over the patient's persistent levels of distress, social avoidance, sense of hopelessness, and increased avolition. The primary care clinician knew you provided telepsychology services and referred the patient to you because of the convenience and flexibility that telepsychology offered the patient, hoping the patient would have a higher likelihood of attending the visit. Upon reviewing the completed intake forms, you note that the patient reported that their sex recorded at birth was female. However, the patient did not complete the questions about their gender identity and pronouns. Your intake forms ask, "What is your legal name?" and "What name would you like me to use in our visits?" The patient notes that their legal name is Samantha and that they would like you to use the name Sam during the visit.

As you log in to your virtual visit platform to meet the patient, you notice that the patient's video is not streaming, but you can hear and speak with the patient over your headphones and microphone. You inform Sam that your preference is to see them over video, but Sam reports their camera is not working and that the wireless internet signal is weak and will cause the call to disconnect if they attempt to turn the video on; you decide to continue the visit. After introducing yourself and providing your pronouns, your credentials, collecting information about the address of their physical location, alternative contact method, describing limits of confidentiality, and asking if Sam has a private space to talk, you ask Sam what pronouns they would prefer you use during your visits. "Oh, you noticed I left that part blank," and continues by stating, "Well, I guess that's part of the reason I'm talking to you. Ever since the pandemic began, I moved back in with my parents and I have had a lot more time to think and to be by myself. I used to be the type of person who would always do things and I would stay busy because I wouldn't have to sit with my thoughts or think about how uncomfortable I feel in my body."

As you visit with Sam, you learn that they have identified as "queer," an umbrella term used by Sam to denote not identifying as a cisgender heterosexual person. Sam discloses they avoid social interactions with others because they worry how they are perceived and that people are confused by their appearance. "People will often use 'ma'am' and 'sir' in the same sentence, and they don't know how to refer to me. Ever since I was young, I have felt like I am male, but others don't view me this way and I hate it." Your attentive, warm, and nonjudgmental and open questions lead Sam to disclose to you that they have been experiencing gender-related distress since age 12. Subsequently, you ask Sam about how video visits play a role in experiencing 
gender-related distress. Sam responds, "Having to look at myself on camera makes me feel really feminine and I don't like that feeling, it's not me."

\section{Telepsychology in the COVID-19 Pandemic}

In early 2020, a worldwide call for physical distancing to reduce COVID-19 transmission led to the rapid uptake of telehealth services (Centers for Disease Control and Prevention, 2020; World Health Organization, 2020). In March 2020, mental health clinicians transitioned from primarily in-person to predominately telepsychology encounters within just two weeks. In the United States, we experienced an unprecedented upswing in telehealth service demand, increasing by $186 \%$ since the onset of COVID-19 (Sammons et al., 2020). For many health service psychologists, the sudden shift to telepsychology preceded formal training in telehealth best practices, let alone best practices in telehealth service delivery for transgender and gender diverse (TGD) populations. A lack of population-specific guidance for TGD increases the risk of further jeopardizing the health and well-being of this already vulnerable population (Cicero et al., 2020).

\section{Transgender and Gender Diverse Populations and the COVID-19 Pandemic}

Despite well-documented health disparities and risk factors for worsened mental health outcomes, the TGD population has received little attention during the COVID-19 pandemic (Gonzales et al., 2020). Historically, TGD individuals have experienced higher rates of multiple chronic health conditions compared to cisgender peers (Downing $\&$ Przedworski, 2018). Such preexisting conditions may exacerbate risks among this group to contract COVID-19. Research conducted during the COVID-19 pandemic reveals an increased report of internalizing symptomatology among TGD individuals, including elevated rates of social withdrawal, anxiety, depression, and loneliness (Flentje et al., 2020). In addition, compared to before the COVID-19 pandemic, TGD individuals have reported significantly reduced access to gender-affirming medical care and increased socioeconomic insecurity (Jarrett et al., 2021).

Given the increased vulnerabilities and health disparities among TGD individuals, strategies to promote genderaffirming care to telepsychology platforms are essential. In this article, we use gender-affirming as an umbrella term that recognizes, acknowledges, and respects an individual's gender identity and gender expression, despite the individual's sex recorded at birth. Providing gender-affirming evidence-based telepsychology services to address preexisting and emergent mental health concerns may mitigate further exacerbation of health disparities.

\section{Telepsychology Services for Transgender and Gender Diverse Individuals}

Longstanding systemic barriers to mental health care among TGD individuals, such as time and financial costs, may be eased via gender-affirming telepsychology. Perhaps the most immediate benefit of providing telepsychology to TGD patients is increased accessibility and physical safety. Patients can now receive care from the comfort and convenience of their home, resulting in less need to take time off work to travel to therapy and other medical appointments. This convenience is true for TGD patients living in rural areas for whom telepsychology services have the potential to expand access to care by decreasing the financial and time burden associated with traveling to see a gender health specialist (Asaad et al., 2020; Koch \& Knutson, 2016; Magnus et al., 2020).

For TGD individuals with adequate access to telepsychology, gender-related stressors to care may still undermine optimal treatment engagement. First, a lack of privacy or safety in a patient's physical environment may prevent TGD individuals from discussing concerns with a health service psychologist. For example, if a patient has not disclosed their gender identity to those within their immediate environment, discussing gender-related topics during a telepsychology visit may not be safe or feasible. Given that telepsychology relies on video and audio functions as a means of interpersonal connection and communication, some TGD individuals may experience gender-related distress related to voice and appearance. Distress stemming from video or audio may thwart active engagement during a telepsychology encounter and contribute to avoidance of telepsychology altogether. Notably, the impact of gender-related distress specific to voice or appearance may become most pronounced when TGD individuals seek to establish care with a new health service psychologist. In such instances, increased preoccupation with one's voice and appearance, fear of invalidation, rejection, or misgendering from an unfamiliar provider may intensify gender-related distress (Sequeira et al., 2021). In the vignette, Sam intentionally left their gender identity and pronouns blank on the intake packet out of concern of the new health service psychologist not understanding or rejecting them if they disclosed.

To promote the delivery and uptake of telepsychology among TGD individuals, we provide recommendations on how health service psychologists can engage in genderaffirming telepsychology services. In addition, this article reviews common concerns observed by health service 
psychologists at a high-volume gender clinic. It provides anticipatory guidance and best practice recommendations in optimizing care for TGD adults receiving telepsychology services in the context of the COVID-19 pandemic. The recommendations provided are intended to build upon foundational guidelines and competencies previously established by the American Psychological Association in the practice of telepsychology (American Psychological Association, 2013).

\section{Clinical and Ethical Challenges}

As noted in the vignette, setting the stage for a genderaffirming telepsychology environment is critical for TGD patients to build trust and comfort. In the vignette, before the initial telepsychology visit, Sam was provided with an intake packet, which asked about legal name, name and pronouns to use during the visit, sex recorded at birth, and gender identity. It is essential to not rely on patient's characteristics (e.g., voice or appearance) to inform name or pronoun use. When starting the initial telepsychology video visit, display your name and pronouns, such as "Sara Johnson, $\mathrm{PhD}$ (she/ her)." The ideal practice is to introduce yourself and state your pronouns verbally (e.g., "My name is Dr. Johnson and I use she/her pronouns"). Next, verbally confirm the patient's affirmed name and pronouns listed on the intake packet. Providing Sam with the intake packet and verbally checking back with Sam about the information on the intake packet, and the health service psychologist's self-disclosure of pronouns, informed Sam that the health service psychologist had an awareness of gender-affirming practices (Loo et al., 2021; Sequeira et al., 2021).

\section{The TGD Patient Lacks Access to Reliable Internet or Video-Capable Device(s)}

Rates of economic insecurity and hardship are significantly higher for TGD people compared to cisgender people (Badgett et al., 2019). We have found that this hardship translates to patients lacking access to reliable internet or video-capable devices, such as a smartphone or laptop. As an alternative to the use of these devices, health service psychologists should offer TGD patients the opportunity to conduct telephone-based visits. While telephone-based visits may pose unique limitations (e.g., reduced perception of interpersonal connectedness or reduced visual cues to assist in interpreting patients' affect), this modality can still benefit patients in distress. Suppose a TGD patient has limited or unreliable access to telephone services. In that case, health service psychologists can help TGD patients identify and pursue available social services or programs that provide no or low-cost access to telecommunication services, which can also help build rapport with TGD patients.
Health service psychologists should continually assess and address patients' financial concerns for reimbursement, particularly when telephone-based applications are used instead of video. Given a quickly evolving healthcare landscape, it is important for health service psychologists, especially those working with TGD patients, to remain current on reimbursement guidelines. This information may include directing TGD patients to appropriate insurance entities and representatives to resolve financial concerns or inquiries. Health service psychologists should be aware of policy changes that impact reimbursement of telepsychology services after the COVID-19 public health emergency (Health Resources and Services Administration, n.d.). These policies and procedures could disproportionately impact TGD patients given the increased healthcare and related financial challenges that they tend to experience (Badgett et al., 2019).

Alternatively, health service psychologists can consider offering face-to-face psychotherapy services to TGD patients who cannot engage in telepsychology. All in-person psychotherapy services should adhere to CDC guidelines and recommendations for vaccination and use of personal protective equipment, including face-covering, eye protection, and physical distancing. If the health service psychologist cannot provide face-to-face or telepsychology services, referrals to gender-affirming clinicians or clinics should offered.

\section{The TGD Patient Lives in A Gender Non-Affirming Environment or Lacks Privacy, Housing, or Utility Services to Engage in Telepsychology Visits}

Recognize that because of privacy limitations, patients' preferences may differ from when in person. Even if the health service psychologist has an established relationship, it is essential not to assume that telehealth is possible for TGD patients. In addition, to reduce the risk of personal safety concerns among TGD patients during telepsychology visits, health service psychologists should ask the patient directly about their level of safety and privacy within their immediate environment. An example inquiry might be, "Are you currently in an environment where you feel comfortable to discuss the topics or concerns that are most important to you today with safety and privacy?"

Health service psychologists should confirm that patients are alone before proceeding with any personal or sensitive information discussions. If it becomes clear that another person is present, health service psychologists should ask TGD patients to identify the person(s) and their relationship to the patient. Sometimes, TGD patients may receive emotional and psychological benefits from the presence of a trusted person or companion. In such instances, providers should engage in a collaborative dialogue with the patient to negotiate what concerns are most appropriate to discuss privately versus topics or concerns that are appropriate to 
discuss in the presence of a support person. Health service psychologists should use their clinical judgment to ensure optimal patient privacy and comfort when a support person is involved in the clinical interaction. Appropriate permissions and written consent should also be obtained by TGD patients before including others in session.

If a TGD patient indicates that their current location or environment is not private or safe, health service psychologists should assist the patient with determining the most appropriate next step. In some circumstances, minor logistical modifications or problem-solving may address privacy concerns. For example, a health service psychologist may inform the TGD patient about headphones to increase privacy. Alternatively, help the TGD patient identify a nearby physical location that offers increased privacy or safety. A provider can also discuss using an agreed-upon "safety word" to communicate if or when a TGD patient's privacy or safety status changes during the telepsychology visit. A safety word can effectively signal to the health service psychologist that a pause in the discussion of sensitive information is needed. If reasonable modifications or problem-solving cannot address TGD patient safety and privacy concerns, health service psychologists should work with the TGD patient to reschedule the telepsychology visit. In such situations, health service psychologists should proactively co-create a plan with the TGD patient to ensure gender-affirming telepsychology services can proceed with fewer barriers.

Incorporating core components of telepsychology is critical to preserving and promoting the well-being of TGD patients and communities during and after the COVID-19 pandemic. While it is beyond the scope of this article to review in detail major aspects of telepsychology, we briefly underscore the importance of its application among TGD patients. First, informed consent procedures represent a valuable opportunity to establish trust, transparency, and rapport with TGD patients. In the context of telepsychology, emphasis on confidentiality and limits to confidentiality, as well as patients' rights (e.g., choice around disclosure of personal information) may be particularly beneficial. We recommend that health service psychologists solicit TGD patients' questions and concerns throughout the informed consent process to increase patient comfort and understanding. Additionally, health service psychologists should ensure that required consent forms (paper and electronic) are inclusive and affirming (e.g., include spaces to record a patient's affirmed name, in addition to legal name, when required). Next, given the increased risk for suicide among TGD individuals and communities, in combination with the physical distance between patients and providers during telepsychology visits, health service psychologists should always conduct an affirming risk assessment that includes an evaluation of suicidal ideation. We strongly recommend that health service psychologists familiarize themselves with clinical procedures and resources for working remotely with patients at risk for harm to self or others (see Zero Suicide Institute, n.d.).

As appropriate, consider prioritizing referral resources or services that are specific to TGD persons, families, or communities to maximize support. Minimize assumptionmaking, however, by collaboratively discussing with TGD patients which options are most preferred (e.g., population-specific versus non-population specific crisis resources and services). Evaluation should also be given to a TGD patient's concern for harm from others in their environment. Affirming practices that promote patient safety and well-being may include setting aside time during early phases of a telepsychology visit to collect emergency information (e.g., document a TGD patient's current physical location, telephone number, and desired emergency contact). Depending upon a TGD patient's disclosure and social transition status, providers may need to clarify what name and pronouns to use if speaking about the patient with others. For example, a provider may inquire, "What name and pronouns are safe to use with this emergency contact?"

In addition, health service psychologists should keep awareness around diagnostic coding for visits and understand the implications this has for the TGD patient's privacy and confidentiality. For example, when working with patients to manage gender dysphoria, health service psychologists will likely submit insurance claims using the diagnosis of "Gender Dysphoria" listed in the Diagnostic and Statistical Manual, Fifth Edition (DSM5; American Psychiatric Association, 2013). However, if the patient is insured as a dependent, in most states, the primary policyholder is mailed an explanation of benefits that will include diagnostic codes that use the International Classification of Disease (ICD; World Health Organization, 2016, 2019). Health service psychologists must recognize that depending on whether the ICD-10 or ICD-11 is used by the insurer, the DSM- 5 code that was submitted to insurance will be translated to an ICD diagnosis of "gender identity disorder in adolescence and adulthood," "transsexualism," or "gender incongruence." Recognize that the diagnosis has implications for your patient's privacy and confidentiality as the person receiving the explanation of benefits will be aware of the diagnostic code used. In addition, the patient may report feeling distress due to the diagnostic label. It is critical for the health service psychologist to have these conversations with the TGD patient and to understand the clinical and ethical implications of a diagnosis on the TGD patient's well-being. 


\section{The TGD Patient Prefers to Engage in Telepsychology Visits With the Camera Turned Off}

Health service psychologists should engage in a compassionate and informed inquiry to clarify a TGD patient's preference to turn off their camera during a telepsychology visit. This assessment can help a provider evaluate if the TGD patient's preference to turn off their camera is related to their gender-related appearance or transition status. To effectively explore this patient concern, health service psychologists can ask open-ended questions about a TGD patient's previous experience in telepsychology. Should it be a TGD patient's first time using telepsychology, health service psychologists should use this opportunity to help the patient become oriented to telepsychology visits, including what to expect during the visit and usual challenges and concerns (e.g., losing audio or visual connection during visit). For returning telepsychology patients, information about their previous experiences (both positive and negative) may help providers to develop a more informed and individualized approach toward the current course of gender-affirming telepsychology.

Next, mental health providers should inquire about any gender-specific contributors to distress within the telepsychology encounter, particularly the occurrence of voice- and/ or appearance-related distress. Gender-related voice-related distress (or "voice dysphoria") occurs among some TGD individuals who feel uncomfortable or dissatisfied with their voice in relation to their affirmed gender identity (Moog \& Sund, 2021). For example, a transmasculine person (e.g., an individual who is recorded female at birth and identifies as male) may experience gender-related voice dysphoria if or when they desire a vocal tone that feels more aligned with their affirmed gender identity. Similarly, gender-related appearance-related distress occurs among some TGD individuals who feel uncomfortable or dissatisfied with a current aspect of their facial or physical appearance in relation to their affirmed gender identity. For example, a transfeminine person (e.g., an individual who was recorded male at birth and identifies as female) may experience gender-related appearance-related distress secondary to undesired and unwanted facial hair. For some TGD patients, the visual components of a telepsychology visit may exacerbate gender-related distress (Sequeira et al., 2021).

For TGD individuals who endorse voice- or appearancerelated distress, health service psychologists should assess whether the TGD patient has identified any gender-affirming strategies that help to reduce or alleviate their distress. For example, some TGD individuals benefit from implementing gender-affirming behavioral interventions that help adapt one's gender expression or appearance in desired ways. Some common examples include vocal training, chest binding, chest padding, use of hairpiece, makeup, shaving, and clothing. In addition to gender-affirming behavioral interventions, some TGD individuals desire and benefit from biomedical (e.g., gender-affirming hormone therapy; hair removal) and surgical interventions (e.g., mastectomy with the creation of masculine chest; breast augmentation) that aim to further modify the body in a manner that affirms one's gender identity. Because biomedical and surgical interventions are often longer-term treatment goals for TGD patients, health service psychologists should focus on identifying behavioral strategies that can be immediately implemented during the telepsychology encounter to help manage or alleviate voice- or appearance-related distress. One initial strategy used in video visits has been to have TGD patients close their video self-view during a telepsychology encounter (Pfund et al., 2020; Warnock-Parkes et al., 2020). It may be appropriate to allow a TGD patient to turn off their camera function entirely in some situations. However, caution is warranted as this strategy may unintentionally perpetuate safety behaviors and reinforce TGD patients' negative self-beliefs and avoidant interpersonal behaviors (Warnock-Parkes et al., 2020), which perpetuates social isolation and loneliness. Based on the severity of a TGD patient's voice- or appearance-related distress, health service psychologists should collaboratively construct treatment goals with patients that target this specific source of distress. Cognitive strategies to challenge gender-based standards and stereotypes may be helpful (e.g., "Do all women have long hair?"). Goals may include increasing distress tolerance and reducing avoidant interpersonal behaviors in a manner that also cultivates self-compassion and self-acceptance. Using evidence-based approaches, such as exposure therapy, may be valuable pathways to promote incremental improvements in engagement in telepsychology among some TGD individuals. All treatment plans, goals, and activities should be collaboratively and compassionately discussed and mutually agreed upon, with a therapeutic rationale provided. If needed, a referral to a health service delivery psychologist with expertise in transgender health can be made by visiting the website for World Professional Association for Transgender Health (https://www.wpath.org).

\section{The TGD Patient is Distressed Because of Limited Access to, or Rescheduling of, Gender-Affirming Biomedical/Surgical Care}

For TGD individuals with scheduled gender-affirming surgeries, the experience of a canceled or rescheduled procedure because of COVID-19 often causes feelings of distress, frustration, and invalidation. Gender-affirming surgeries are considered medically necessary versus "elective" in nature. For this reason, it is essential for health service psychologists to acknowledge and validate feelings of distress associated with limited access to, or postponement of, biomedical 
and surgical care among TGD individuals. Health service psychologists can establish collaborative working plans that provide a TGD patient with an increased sense of direction. Validation can be provided by explicitly reaffirming the amount of effort and time a patient has put into preparing and planning for gender-affirming biomedical/surgical procedures and interventions. Health service psychologists should partner with TGD patients to identify and bolster resilience factors that promote sustained coping and management of gender dysphoria in the context of biomedical or surgical postponement.

In some circumstances, it may be essential to provide TGD patients with additional gender-care-related anticipatory guidance or referrals to facilitate continuity of gender care during the COVID-19 pandemic. For example, in the United States, testosterone (used to masculinize secondary sex characteristics) is listed in Schedule III of the Controlled Substance Act. In the COVID-19 State of Emergency waivers, most states allow for prescriptions for controlled substances to patients without an in-person medical evaluation; however, not all prescribers will feel comfortable with this. Despite the COVID-19 State of Emergency's waiver on telehealth practices across the United States, this waiver may end and consequently may disrupt the continuity of medical care a patient receives. Anticipating TGD population-specific barriers, discussing these concerns with TGD patients, and providing an up-to-date referral list to gender-affirming medical and behavioral health providers may promote gender-related health and well-being. To minimize disruptions to telepsychology once the COVID-19 State of Emergency waiver expires, health service psychologists could consider applying for the Psychology Interjurisdictional Compact (PSYPACT; https://psypact.site-ym.com).

\section{The TGD Patient Expresses Uncertainty About How to Maintain Their Health and Well-Being During the COVID-19 Pandemic}

To maximize TGD patient health and safety, health service psychologists should assess a patient's health literacy and assumption about the understanding of the COVID-19 virus. Health service psychologists should also encourage adherence to CDC recommendations for mask wearing and physical distancing during the COVID-19 pandemic. As needed, providers can provide basic psychoeducation and referral to a licensed medical professional regarding the vaccination, assessment, and treatment of COVID-19. When needed, providers can also assist TGD individuals in managing any psychological factors (e.g., fear, anxiety, mistrust) that reduce their engagement in recommended preventative services. Given the increased prevalence of adverse health outcomes among TGD people compared to cisgender people, health service psychologists should encourage patients to seek preventative health screenings and care to avoid future health issues or complications.

In some instances, TGD patients may endorse misconceptions related to the COVID-19 virus. For example, the authors have observed situations where TGD patients living with HIV (Human Immunodeficiency Virus) or patients prescribed HIV pre-exposure prophylaxis erroneously assumed protection and immunity against the COVID-19 virus due to their use of an antiretroviral medication. Health service psychologists should immediately correct such assumptions to reduce patient risk. Providers should also keep in mind that rates of sexually transmitted infections (STIs) have increased during the COVID-19 pandemic. As appropriate to a TGD patient's sexual health history and behaviors, providers should inform TGD patients about HIV and STI harm-reduction strategies (Gravett \& Marrazzo, 2021), including the availability of pre-exposure prophylaxis for HIV (https://preplocator.org).

\section{Providing Gender-Affirming Telepsychology Services}

The introductory clinical vignette with Sam illustrates a gender-affirming approach to telepsychology service delivery that helped ascertain the patient's experience of gender invalidation (e.g., misgendering) and gender-related appearance distress. This approach can help facilitate the development of rapport, as Sam may feel more deeply seen and understood by the clinician. Although this approach can be helpful and validating, it does not describe how cognitive and behavioral skills can mitigate the effects of genderrelated stigma and distress. Hence, creating opportunities to explore how these factors impact TGD patients and their self-management behaviors can assist the clinician in contextualizing presenting concerns and behaviors that may limit treatment engagement in the long term (e.g., keeping the camera off). This may facilitate a therapeutic relationship in which the TGD patient is willing to name their stressors and generate expanded coping repertories for addressing them (e.g., exposure practices, initiating gender-affirming treatment).

A response that displays familiarity with gender-affirmative practices can include a question that prompts Sam to reflect on how gender-related stigma and distress undermine their daily functioning and overall well-being. The goal here is to create opportunities to introduce, explain, and validate the functional impact of gender-related distress and oppression in therapy. For instance, the clinician can respond by saying,

"Sam, I hear you say that it drains you to be misgendered so frequently and so intensely in different set- 
tings. I'm curious to know about your experiences of gender invalidation and how they affect your day-today life."

Following this exchange, the health service psychologist can continue welcoming Sam to explore and reflect on the impact of gender-related stressors by asking, "How might these experiences influence the appearance-related anxiety in therapy ... and does it affect your sense of connection from those around you, even your loved ones?"

In the therapeutic exchange, Sam may open up about their experiences of gender-related appearance anxiety and difficulties related to their gender expression. For instance, "My parents and I fought the last time I buzzed my hair." In this exchange, clinicians may incorrectly attribute this statement to Sam's desire to be autonomous or rebellious instead of affirming or validating Sam's experience of gender invalidation. A more effective approach could be to help Sam reflect on how gender-related emotional distress limits their ability to trust and connect with others. Such statements can help encourage values-based living and adaptive coping before, during, and after one's engagement in gender transition-related services. For instance, the clinician can respond by saying,

"It makes sense that you have to present a certain way
to maintain your safety at home... I imagine it's very
painful to live that and to see yourself on camera...
Perhaps this is something we can continue exploring in
therapy? Together, we can explore options for gender
care, if that's something you're interested in. We can
also discuss strategies you can use to manage your
current discomfort with appearance. This may help
you engage more meaningfully with your chosen loved
ones and health providers you meet along the way."

\section{Conclusion}

This article demonstrates that gender-affirming practices can be comparable for in-person and telepsychology delivery. Both modalities seek to reduce distress symptoms (Backhaus et al., 2012; Hilty et al., 2013) despite facing similar barriers, such as concerns related to privacy and stigma. For transgender and gender diverse patients, it can be surmised that barriers related to resource instability, fear of gender invalidation or mistreatment, and safety concerns (e.g., the threat of violence) can limit treatment engagement across the two modalities (James et al., 2016). It is also essential to highlight the telehealth-specific factors that may limit a gender diverse patient's participation in remote services, such as limited technology access, poor access to quality telehealth services, or gender-related distress (van der Miesen et al., 2020; Green et al., 2020). Indeed, navigating telehealth platforms involves facing experiences that may trigger gender dysphoria, such as seeing one's on-screen video.

These limitations imply that additional preparation and focus on TGD patient experience in treatment may be helpful in services delivered remotely. In both modalities, health service psychologists can deliver evidence-based practices (e.g., validation, exposure practice) in a manner that communicates respect and familiarity with TGD communities. Health service psychologists should follow established guidelines, such as those published by the American Psychological Association, to deliver gender-affirming psychological care (APA, 2015) and to practice telepsychology more broadly (APA, 2013). Both guidelines can be used to select the appropriate technology and to implement gender-affirming practices within that platform (e.g., when, where, and how to display affirmed names and pronouns). Emerging evidence suggests that TGD youth are more willing to participate in telehealth treatment if providers indicate regular participation in training about transgender health (Sequeira et al., 2021). Video conferencing has made it more convenient for health service psychologists to seek training and consultation to promote competency in telepsychology and gender-affirming practices.

The COVID-19 pandemic has expedited the integration and implementation of telepsychology, which few health service psychologists were prepared for before 2020. While the rapid adoption of this service modality is fraught with systems- and provider-level challenges, the significance of its impact on the TGD community cannot be understated. TGD individuals remain among those at most significant risk for mental health problems during the COVID-19 pandemic. Through thoughtful consideration and implementation of specific gender-affirming evidence-based practices, our current public health crisis provides an invaluable opportunity to optimize the overall health and well-being of the TGD population. We hope this article encourages health service psychologists to deliberately practice genderaffirming telepsychology to provide culturally responsive and patient-centered care during the COVID-19 pandemic and beyond.

\section{Key Clinical Considerations}

1. Set the stage for a gender-affirming telepsychology environment by (a) including gender-related questions in the intake packet, (b) including your pronouns by your name, and (c) being aware about and addressing genderrelated distress related to appearance or voice.

2. Assess socioeconomic needs and refer to social services or resources. 
a. If the patient has limited access to phone service, consider the Lifeline Program for Low-Income Consumers: https://www.fcc.gov/general/lifeline-progr am-low-income-consumers

b. Provide information on state and local housing/ rental/utility assistance: https://nlihc.org/rental-assis tance

3. Assure that referrals made to the patient are to genderaffirming clinicians and support.
a. World Professional Association for Transgender Health: https://www.wpath.org/provider/search
b. GLMA Health Professionals Advancing LGBT + Equality: http://www.glma.org
c. LGBT + Community Center Directory: https://www. lgbtcenters.org/LGBTCenters

4. Recognize the unique biomedical and psychosocial issues TGD patients face, such as rescheduling of gender-affirming surgeries because of COVID- 19 .

5. Provide information on gender-affirming crisis hotlines, as clinically relevant:

a. Trans Lifeline: https://translifeline.org or (877) $565-8860$

b. The Trevor Project: https://www.thetrevorproject. org or (866) 488-7386

Funding There are no funding sources to disclose.

\section{Declarations}

Disclosures None.

\section{References}

American Psychiatric Association. (2013). Diagnostic and statistical manual of mental disorders ( $5^{\text {th }}$ ed.). https://doi.org/10.1176/appi. books.9780890425596

American Psychological Association. (2015). Guidelines for psychological practice with transgender and gender nonconforming people. American Psychologist, 70(9), 832-864. https://doi.org/10. 1037/a0039906

American Psychological Association. (2013). Guidelines for the practice of telepsychology. Retrieved on July 23, 2021, from https:// www.apa.org/practice/guidelines/telepsychology

Asaad, M., Rajesh, A., Vyas, K., \& Morrison, S. D. (2020). Telemedicine in transgender care: A twenty-first-century beckoning. Plastic and Reconstructive Surgery, 146(1), 108e-109e. https://doi.org/ 10.1097/PRS.0000000000006935

Cicero, E. C., Reisner, S. L., Merwin, E. I., Humphreys, J. C., \& Silva, S. G. (2020). The health status of transgender and gender nonbinary adults in the United States. PloS One, 15(2), e0228765. https://doi.org/10.1371/journal.pone.0228765
Backhaus, A., Agha, Z., Maglione, M. L., Repp, A., Ross, B., Zuest, D., ... \& Thorp, S. R. (2012). Videoconferencing psychotherapy: a systematic review. Psychological services, 9(2), 111.https:// doi.org/10.1037/a0027924

Badgett, M. L., Choi, S. K., \& Wilson, B. D. (2019). LGBT poverty in the United States. Los Angeles, CA: The Williams Institute and American Foundation for Suicide.

Centers for Disease Control and Prevention. (2020). Coronavirus disease 2019 (COVID-19): How to protect yourself \& others. Retrieved on August 3, 2021, from https://www.cdc.gov/coron avirus/2019-ncov/prevent-getting-sick/prevention.html

Downing, J. M., \& Przedworski, J. M. (2018). Health of transgender adults in the US, 2014-2016. American Journal of Preventive Medicine, 55(3), 336-344. https://doi.org/10.1016/j.amepre. 2018.04.045

Flentje, A., Obedin-Maliver, J., Lubensky, M. E., Dastur, Z., Neilands, T., \& Lunn, M. R. (2020). Depression and anxiety changes among sexual and gender minority people coinciding with onset of COVID-19 pandemic. Journal of General Internal Medicine, 35(9), 2788-2790. https://doi.org/10.1007/ s11606-020-05970-4

Gonzales, G., de Mola, E. L., Gavulic, K. A., McKay, T., \& Purcell, C. (2020). Mental health needs among lesbian, gay, bisexual, and transgender college students during the COVID-19 pandemic. Journal of Adolescent Health, 67(5), 645-648. https://doi.org/ 10.1016/j.jadohealth.2020.08.006

Gravett, R. M., \& Marrazzo, J. M. (2021). HIV and COVID-19: Lessons From HIV and STI Harm Reduction Strategies. Current HIV/AIDS reports, 18(4), 261-270. https://doi.org/10.1007/ s11904-021-00562-1

Green, A.E., Price-Feeney, M. \& Dorison, S.H. (2020). Implications of COVID-19 for LGBTQ Youth Mental Health and Suicide Prevention. New York, New York: The Trevor Project. https://www. thetrevorproject.org/wp-content/uploads/2021/08/Implications-ofCOVID-19-for-LGBTQ-Youth-Mental-Health-and-Suicide-Preve ntion.pdf

Health Resources and Services Administration. (n.d.). Billing for telebehavioral health. Retrieved on September 23, 2021, from https:// telehealth.hhs.gov/providers/telehealth-for-behavioral-health/billi ng-for-telebehavioral-health/

Hilty, D. M., Ferrer, D. C., Parish, M. B., Johnston, B., Callahan, E. J., \& Yellowlees, P. M. (2013). The effectiveness of telemental health: a 2013 review. Telemedicine and e-Health, 19(6), 444-454. https://doi.org/10.1089/tmj.2013.0075

James, S., Herman, J., Rankin, S., Keisling, M., Mottet, L., \& Anafi, M. A. (2016). The report of the 2015 US Transgender Survey. Washington, DC: National Center for Transgender Equality. https:// transequality.org/sites/default/files/docs/usts/USTS-Full-ReportDec17.pdf

Jarrett, B. A., Peitzmeier, S. M., Restar, A., Adamson, T., Howell, S., Baral, S., \& Beckham, S. W. (2021). Gender-affirming care, mental health, and economic stability in the time of COVID-19: A multi-national, cross-sectional study of transgender and nonbinary people. PloS ONE, 16(7), e0254215. https://doi.org/10.1371/journ al.pone. 0254215

Koch, J. M., \& Knutson, D. (2016). Transgender clients in rural areas and small towns. Journal of Rural Mental Health, 40(3-4), 154. https://doi.org/10.1037/rmh0000056

Loo, S., Almazan, A. N., Vedilago, V., Stott, B., Reisner, S. L., \& Keuroghlian, A. S. (2021). Understanding community member and health care professional perspectives on gender-affirming care-A qualitative study. PloS ONE, 16(8), e0255568. https:// doi.org/10.1371/journal.pone.0255568

Magnus, M., Edwards, E., Dright, A., Gilliam, L., Brown, A., Levy, M., Sikka, N., Siegel, M., Criss, V., \& Watson, C. C. (2020). Development of a telehealth intervention to promote care-seeking among 
transgender women of color in Washington, DC. Public Health Nursing, 37(2), 262-271. https://doi.org/10.1111/phn.12709

Moog, D., \& Sund, L. T. (2021). Clinician and Consumer Perspectives on Gender-Affirming Voice Services. Journal of Voice. https://doi. org/10.1016/j.jvoice.2021.05.009

Pfund, G. N., Hill, P. L., \& Harriger, J. (2020). Video chatting and appearance satisfaction during COVID-19: Appearance comparisons and self-objectification as moderators. International Journal of Eating Disorders, 53(12), 2038-2043. https://doi.org/10.1002/ eat. 23393

Sammons, M. T., VandenBos, G. R., \& Martin, J. N. (2020). Psychological practice and the COVID-19 crisis: A rapid response survey. Journal of Health Service Psychology, 46(2), 51-57. https:// doi.org/10.1007/s42843-020-00013-2

Sequeira, G. M., Kidd, K. M., Coulter, R., Miller, E., Fortenberry, D., Garofalo, R., Richardson, L. P., \& Ray, K. N. (2021). Transgender Youths' Perspectives on Telehealth for Delivery of Gender-Affirming Care. The Journal of adolescent health : official publication of the Society for Adolescent Medicine, 68(6), 1207-1210. https:// doi.org/10.1016/j.jadohealth.2020.08.028

van der Miesen, A. I., Raaijmakers, D., \& van de Grift, T. C. (2020). "You have to wait a little longer": Transgender (mental) health at risk as a consequence of deferring gender-affirming treatments during COVID-19. Archives of Sexual Behavior, 49, 1395-1399. https://doi.org/10.1007/s10508-020-01754-3

Warnock-Parkes, E., Wild, J., Thew, G. R., Kerr, A., Grey, N., Stott, R., ... \& Clark, D. M. (2020). Treating social anxiety disorder remotely with cognitive therapy. The Cognitive Behaviour Therapist, 13.https://doi.org/10.1017/S1754470X2000032X

World Health Organization. (2020). Coronavirus disease (COVID-19) advice for the public. Retrieved on September 23, 2021, from https://www.who.int/emergencies/diseases/novel-coronavirus2019/advice-for-public

World Health Organization. (2019). International statistical classification of diseases and related health problems (11th ed.). https:// icd.who.int/

World Health Organization. (2016). International statistical classification of diseases and related health problems (10th ed.). https://icd. who.int/browse10/2016/en

Zero Suicide Institute. (n.d.). Telehealth and suicide care during the COVID-19 pandemic. Retrieved on September 23, 2021, from http://zerosuicide.edc.org/sites/default/files/Telehealth\%20and\% 20Suicide\%20Care\%20During\%20the\%20COVID-19\%20Pan demic.pdf
Tyson L. Pankey, PhD, MPH (he/him) is a licensed psychologist and assistant professor in the Department of Psychiatry \& Behavioral Sciences at Duke University School of Medicine. He completed his PhD in Counseling Psychology from the University of Wisconsin-Madison. He completed fellowship training at Mayo Clinic with dual specializations in transgender health and family medicine residency education. Dr. Pankey has expertise in culturally responsive behavioral health and education among underserved populations, including sexual and gender diverse individuals.

Dagoberto Heredia Jr, PhD (he/him) is a licensed psychologist and assistant professor of psychology at Mayo Clinic's College of Medicine and Science. His clinical appointments are in the Adult Transitions Program, where he serves as clinical director, and the Transgender and Intersex Specialty Care Clinic. He has served as co-chair of the National Latinx Psychological Association's Orgullo Latinx: Sexual Orientation and Gender Diversity special interest group since 2014.

Jennifer A. Vencill, PhD, ABPP (she/her) is an assistant professor, board-certified clinical health psychologist, and AASECT-certified sex therapist at the Mayo Clinic in Rochester, Minnesota, USA. She practices primarily in Mayo Clinic's Menopause and Women's Sexual Health Clinic and Transgender and Intersex Specialty Care Clinic. Dr. Vencill's research focuses on sexual health, mixed-orientation relationships, and health disparities and minority stress in marginalized sexual and gender communities.

Cesar A. Gonzalez, PhD, LP, ABPP (he/they) is an assistant professor of psychology and family medicine; he is board-certified in clinical psychology. He is certified in the Standards of Care by the World Professional Association for Transgender Health. He serves as the clinical director of Mayo Clinic's Transgender and Intersex Specialty Care Clinic and is an associate program director of the Family Medicine Residency Program. 